\title{
Aobótica de exploración, un mundo de posibilidades
}

\section{Robotic exploration, a world of possibilities}

Yeison Alejandro Aldana ${ }^{1}$

()...

Recepción: 07/10/2020
Aprobación: 27/10/2020
(2)

Publicación: 18/12/2020

\section{Para citar este artículo:}

Aldana, Y. A. (2020). Robótica de exploración, un mundo posibilidades.

Indagare, (8), 227-234. https://doi.org/10.35707/indagare/820

\section{@() $(\Theta \Theta$}

${ }^{1}$ Grupo de investigación D+TEC, Universidad de Ibagué, Colombia. Correo electrónico: yeison.aldana@unibague.edu.co 


\title{
Resumen
}

Los robots, en su mayoría, se han diseñado con la intención de imitar las capacidades del ser humano, ya sea para realizar tareas de manera más ágil o mejorada y con habilidades que las personas no poseen. Este artículo presenta una mirada al desarrollo de un prototipo de robot semiasistido con capacidades mecánicas, de software y de accionamiento que puede ser operado a distancia a través de internet.

\begin{abstract}
Robots mostly have been designed with the intention of imitating the capabilities of human beings, either to perform tasks in a more agile or improved way and with skills that people do not possess. This article presents a glance at the development of a semiassisted robot prototype with mechanical, software and drive capabilities that can be operated remotely via internet.
\end{abstract}

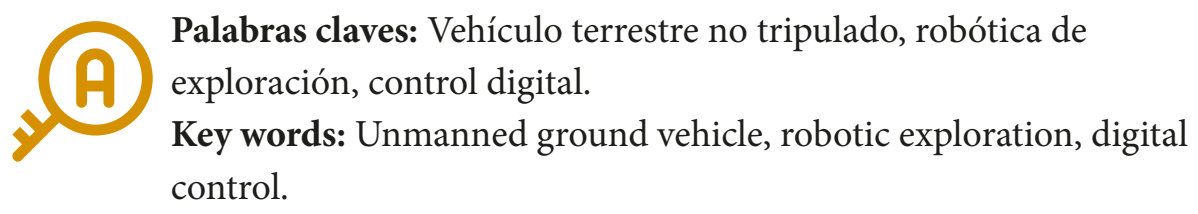

\section{Introducción}

La posibilidad de controlar un robot a largas distancias desde hace algunos años ha dejado de ser un sueño para convertiste en una realidad. El mejoramiento de la capacidad y velocidad del internet abrió muchas puertas para la robótica asistida, además de nuevos entornos de programación como Robot Operating System o ROS (por sus siglas en inglés). Los robots han tomado un papel importante no solo en labores industriales, para las cuales fueron usados en un comienzo en la revolución industrial, como la agricultura o la construcción, sino también en tareas de gran valor como rescate de personas en situaciones de calamidad de difícil acceso, exploración de terrenos peligrosos para el ser humano, extinción de incendios, desactivación de explosivos, etc. (Sánchez, 2020).

Estos robots en la mayoría de los casos son de tipo móvil, puesto que para llevar a cabo sus labores requieren operar con más libertad. Por lo tanto, deben enfrentarse a algunos retos como las adversidades de diferentes tipos de terreno. Y así como los humanos, para los robots es importante poseer sentidos que les ofrezcan información del entorno. En su caso los sensores son de vital importancia para una correcta operación, claro está que estos dependerán de la aplicación específica por desarrollarse (Metz \& Griffith, 2020). 
INDAGA3E e-ISSN: 2357-5042 • Número 8 (2020) • Universidad de Ibagué • doi: https://doi.org/10.35707/indagare/820

Los estudiantes de Ingeniería Electrónica de la Universidad de Ibagué, Nickson Eduardo García Hernández y Cristian Giovanny Molina Hernández, pertenecientes al grupo D+TEC desarrollaron en 2019 un proyecto en el cual explican de manera detallada la metodología para crear un prototipo de robot semiautónomo con capacidad para explorar el entorno y transmitir la información captada por sus sensores a través de internet, si es a largas distancias, o sin su uso si es lo contrario. Además, consta de un brazo robótico que tiene la capacidad de operar de forma automática o manual.

Este prototipo cuenta con subsistemas de percepción, comunicación y acción, que, trabajando en conjunto, logran ofrecer trayectorias estimadas del recorrido del robot. Como se está midiendo la velocidad en todo momento, es posible afrontar obstáculos o variaciones de terreno sin demasiada interacción del piloto, que facilitan su manejo y operación.

\section{Características mecánicas}

El robot se construyó con una arquitectura six wheel steering, que se caracteriza por tener seis ruedas con seis motores, que ofrecen la capacidad para afrontar diferentes tipos de entornos con equilibrio. El brazo robótico cuenta con seis grados de libertad, que le dan gran versatilidad a esta herramienta. El prototipo tiene un peso total de $20 \mathrm{~kg}$ y un tamaño de $0.7 \mathrm{~m} \times 0.5 \mathrm{~m} \times 0.8 \mathrm{~m}$, además cuenta con un sistema de suspensión tipo rocker-bogie (García Hernández \& Molina Hernández, 2019).

Otra característica importante es su autonomía, es decir el tiempo en el que puede operar sin recibir una nueva carga de energía que para este proyecto en particular es de tres horas sobre terrenos medianamente irregulares. Los diseños de las partes del prototipo fueron elaborados en el software de diseño mecánico SolidWorks como se muestra en la Figura 1. 
Figura 1. Diseño del robot móvil en el software SolidWorks

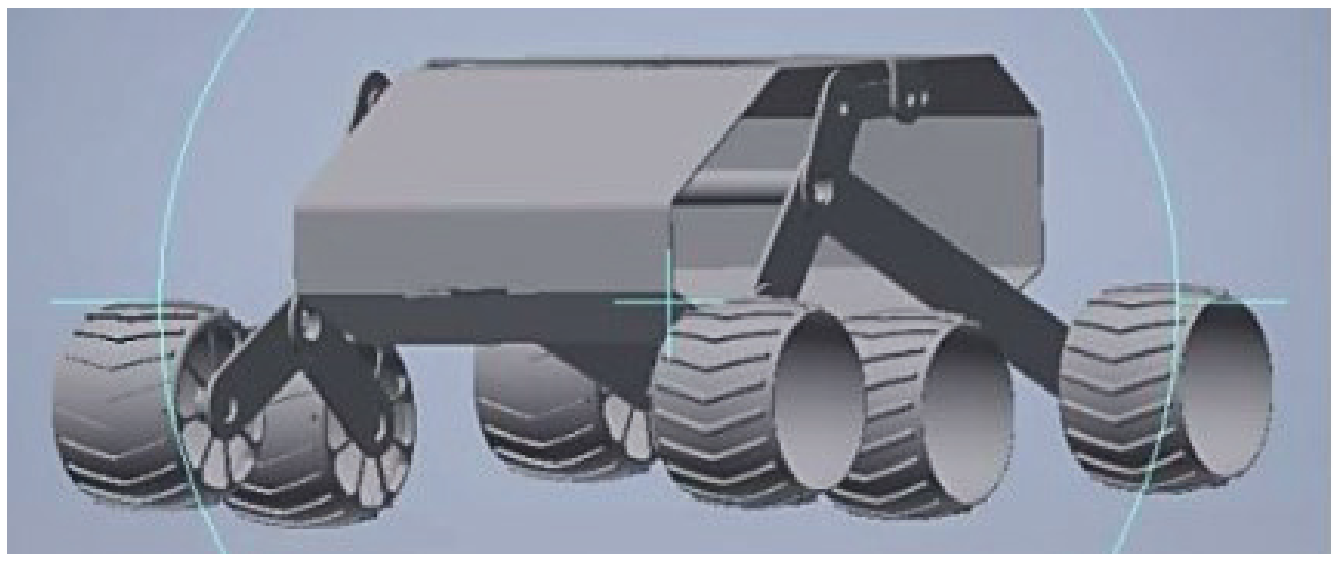

Fuente: García Hernández \& Molina Hernández, 2019

\section{Alimentación o fuente de energía}

Luego de haber definido las características mecánicas, se buscó un sistema de alimentación capaz de suplir las necesidades de los motores y demás dispositivos que tuviesen consumo, por lo tanto, se eligieron dos baterías de 12 voltios a 6 amperios. Debido a que no todos los dispositivos operan a los mismos niveles de corriente y voltaje, se debió realizar una etapa de acondicionamiento de las señales.

\section{Características de software}

El manejo del robot se llevó a cabo a través de un joystick o control de una consola de videojuegos, como se observa en de la Figura 2a, dado que al conectarse a una computadora es posible manejar las diferentes funciones del robot. Para lograr la visión del terreno por explorar fueron usadas una cámara de navegación o webcam común (Figura 2b), y un kinect, Figura 2c, que es un accesorio de consola de videojuegos, y tiene dentro una cámara $3 \mathrm{D}$, una cámara infrarroja, entre otros sensores. La cámara 3D ofrecía al piloto la sensación de profundidad y la cámara infrarroja la capacidad de visión nocturna, característica que no posee una cámara normal o webcam (García Hernández \& Molina Hernández, 2019). 
Figura 2. Herramientas de manejo y visión del robot a) joystick b) cámara web c) kinect

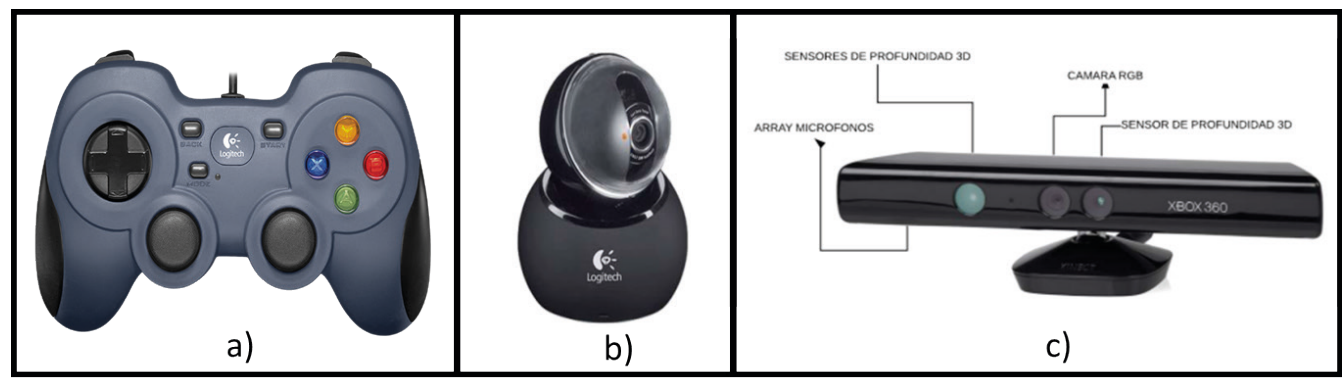

Fuente: García \& Hernández, 2019

\section{Trazado de ruta}

Además del uso de estas cámaras, se implementó un trazado de trayectoria que consistía en obtener la información de los enconders o sensores de las ruedas y así realizar un bosquejo de la ruta dejada conforme avanza, como se muestra en la Figura 3. Esto le brinda al piloto una orientación extra del terreno recorrido y de la ubicación actual, información de vital importancia para este tipo de robot de exploración.

\section{Características de comunicación}

La comunicación a larga distancia, como se mencionó anteriormente, se realizó a través de internet, mediante dos routers, uno en la computadora donde se encuentra el piloto y otro ubicado en la unidad de control del robot. Se realizaron diferentes pruebas evaluando distintos terrenos, distancias en las que se llevaba a cabo la comunicación, entre ellas una prueba realizada con el robot ubicado en el desierto de la Tatacoa y manejado por el piloto desde la ciudad de Ibagué, a $134 \mathrm{~km}$ aproximadamente. A pesar de existir un pequeño retraso por la distancia, se desarrollaron las pruebas con éxito, como se muestra en la Figura 4. 
Figura 3. Trazado de ruta del robot

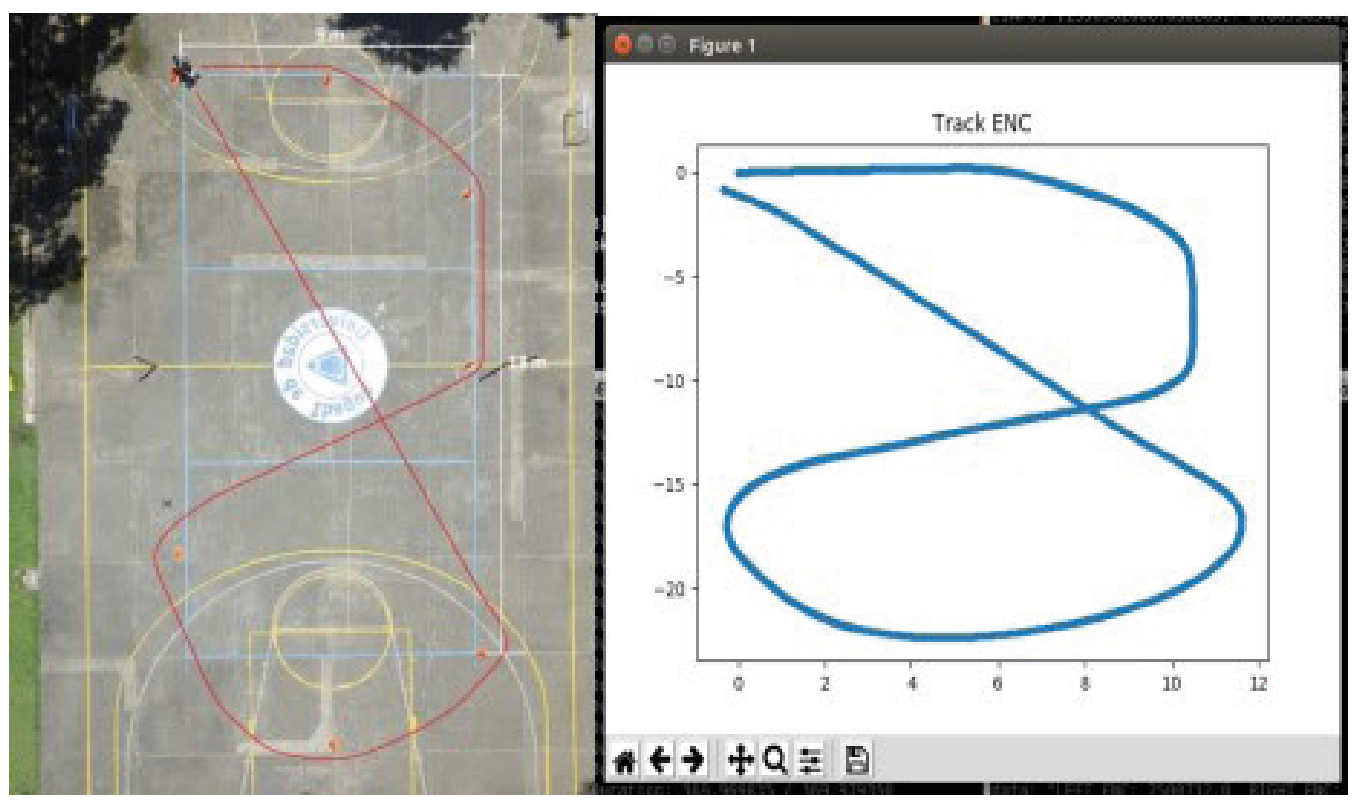

Fuente: García \& Hernández, 2019

Figura 4. Prueba de exploración en el desierto de la Tatacoa

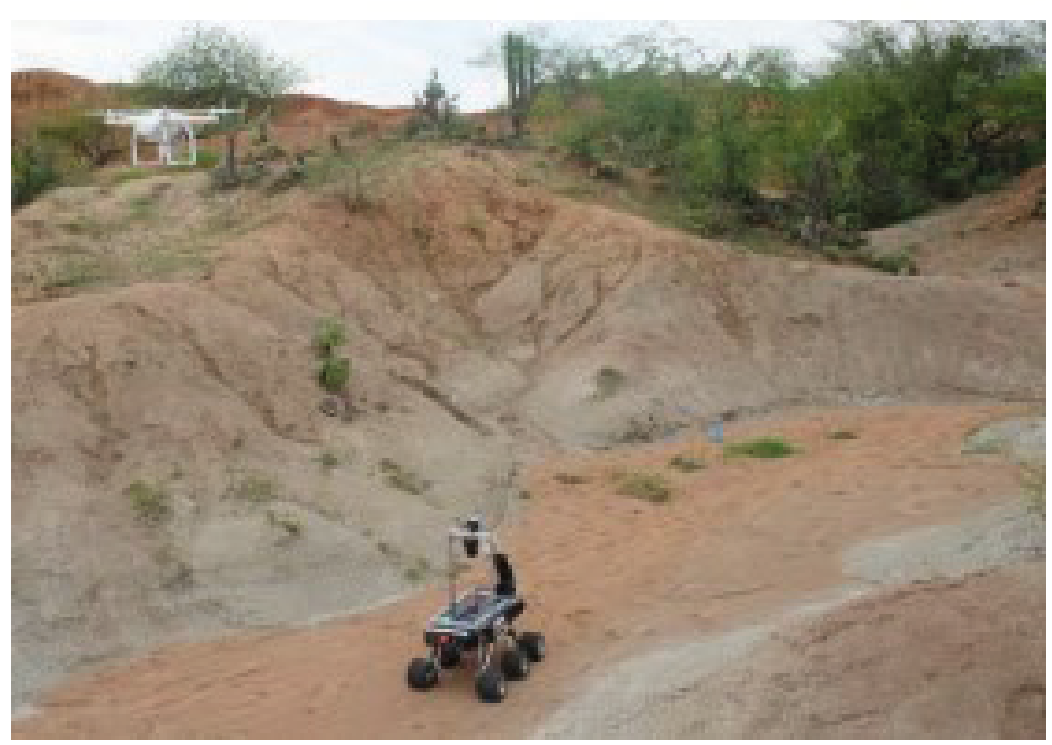

Fuente: García \& Hernández, 2019 
"A pesar de que este es un proyecto exclusivamente educativo o investigativo, con la inversión adecuada puede llegar a ser usado en una de las muchas aplicaciones en las que los robots son no solo una opción, sino una necesidad" indica el investigador Nickson García.

Figura 5. Investigadores desarrolladores del prototipo

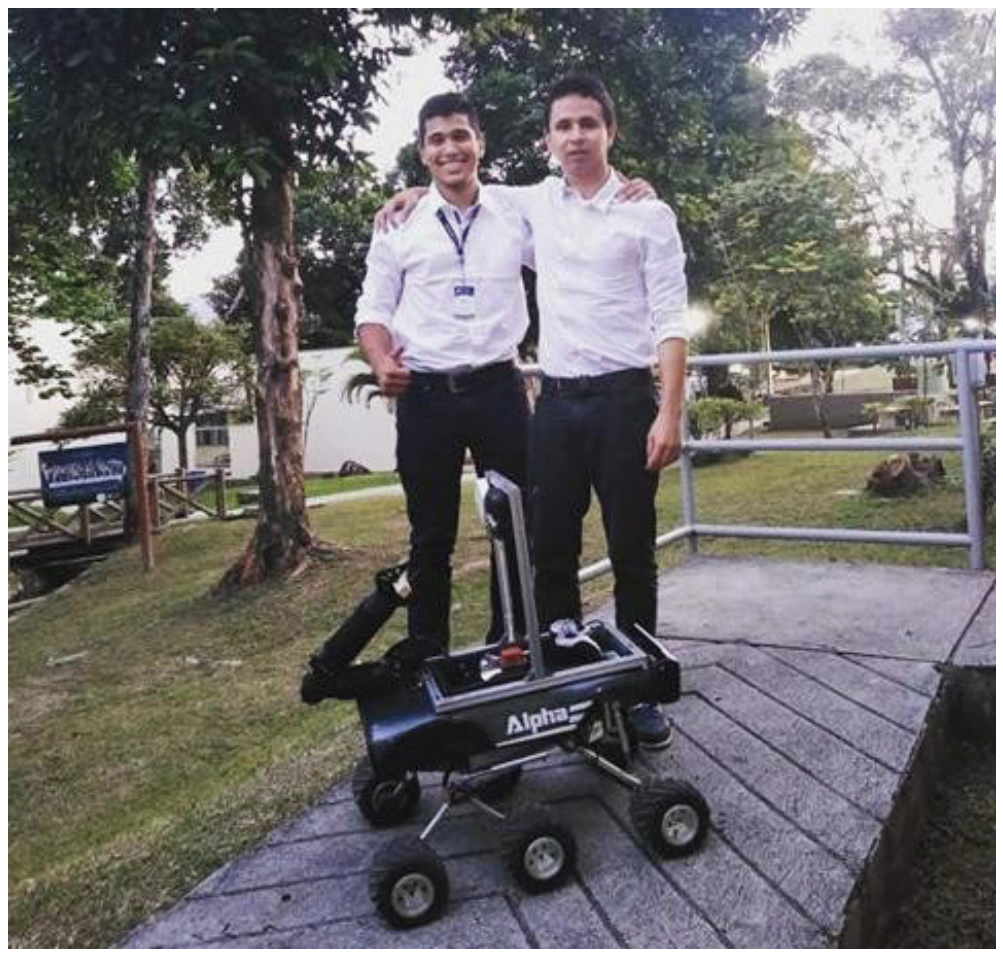

Fuente: autor

\section{Conclusión}

La capacidad de operar robots a largas distancias es una opción que hoy en día posibilita que otras aplicaciones puedan desarrollarse sin riesgo. Además, que la capacidad de cumplir con diferentes labores sin la necesidad de trasladarse hasta el lugar de operación, permite el ahorro de tiempo y costos de transporte u operación. 


\section{Referencias}

García Hernández, N. E., \& Molina Hernández, C. G. (2019). Desarrollo de un robot móvil terrestre semiautónomo con acceso remoto (Trabajo de grado programa Ingeniería Electrónica, Universidad de Ibagué, Tolima, Colombia). Recuperado de https://hdl.handle.net/20.500.12313/1296

Metz, C., \& Griffith, E. (21 de mayo de 2020). Los robots recorren una ciudad cerrada por el coronavirus. The New York Times. Recuperado de https://nyti.ms/32Q1VhM

Sánchez, J. M. (01 de septiembre de 2020). Drones, robots y aplicaciones: tecnologías al servicio de la lucha contra el coronavirus. $A B C$. Recuperado de https://bit.ly/3lC2yCX 